\title{
In vitro production of ergothioneine isotopologues
}

\author{
Mariia A. Beliaeva ${ }^{1}$ Reto Burn ${ }^{1}$, David Lim ${ }^{1}$, and Florian P. Seebeck ${ }^{1 *}$ \\ ${ }^{1}$ Department of Chemistry, University of Basel, Mattenstrasse 24a, Basel 4002, Switzerland
}

\begin{abstract}
Ergothioneine is an emerging component of the redox homeostasis system in human cells and in microbial pathogens, such as Mycobacterium tuberculosis or Burkholderia pseudomallei. Synthesis of stable isotope labelled ergothioneine derivatives may provide important tools for deciphering the distribution, function and metabolism of this compound in vivo. We describe a general protocol for the production of ergothioneine isotopologues with programmable ${ }^{2} \mathrm{H},{ }^{15} \mathrm{~N}^{13} \mathrm{C}$, ${ }^{34} \mathrm{~S}$ and ${ }^{33} \mathrm{~S}$ isotope labelling patterns. This enzyme-based approach makes efficient use of commercial isotope reagents and is also directly applicable for the synthesis of radio-isotopologues.
\end{abstract}

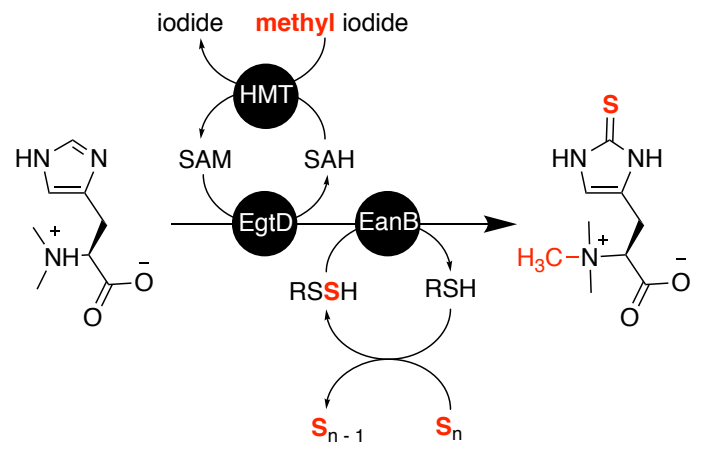




\section{INTRODUCTION}

Ergothioneine (1, Figure 1), is increasingly recognized as an important factor in cellular redox homeostasis. ${ }^{[1]}$ In the last fifteen years, a number of discoveries have shown that ergothioneine is ubiquitous in cellular live, and may participate in a broad range of biochemical processes. First indications that ergothioneine may be an active participant in human physiology came from the identification of a specific ergothioneine transporter (hETT) ${ }^{[2]}$ Discovery of ergothioneine biosynthetic enzymes showed that this simple metabolite is produced by a wide variety of bacteria and almost all fungi, living under a broad range of conditions. ${ }^{[3]}$ Studies with pathogenic bacteria, such as Mycobacterium tuberculosis showed that ergothioneine is essential for survival in their mammalian host. ${ }^{[4]}$ The finding that ergothioneine participates as a cofactor in the biosynthesis of the antibiotic lincomycin A provided a first example of proteins that interact with ergothioneine to elicit biochemical function. ${ }^{[5]}$ Most plants and animals do not produce ergothioneine and rely on supply from their food or environment. While ergothioneine is efficiently retained in the body, metabolized derivatives, such as $\mathrm{N} \alpha$-trimethyl histidine (TMH) or S-methyl ergothioneine are secreted via the kidneys. ${ }^{[6]}$ Oxidized forms of ergothioneine may be recycled by uncatalyzed reactions with glutathione. ${ }^{[7]}$ Identification and characterization of enzymes such as ergothionase and thiourocanate hydratase revealed that ergothioneine degradation may also occur in catalyzed and oxygen-independent processes ${ }^{[8]}$ Bacterial species containing these enzymes are well represented in the gut microbiome, highlighting a possible connection between bacterial activity and the availability of nutritional ergothioneine for humans. ${ }^{[8 b]}$ Despite these advances, our understanding of the physiological role of ergothioneine in microorganisms, in plants and in humans is far from complete. New methodologies may be needed to address this complex biological question with chemical precision.

Figure 1. Top: Focus areas in ergothioneine related research. Bottom: One-pot synthesis of N $\alpha$-trimethylhistidine (TMH, 2) and ergothioneine (1) from $\mathrm{N} \alpha$-dimethylhistidine catalyzed by the halide methyltransferase HMT, the SAM-dependent methyltransferase EgtD and the sulfur transferase EanB.
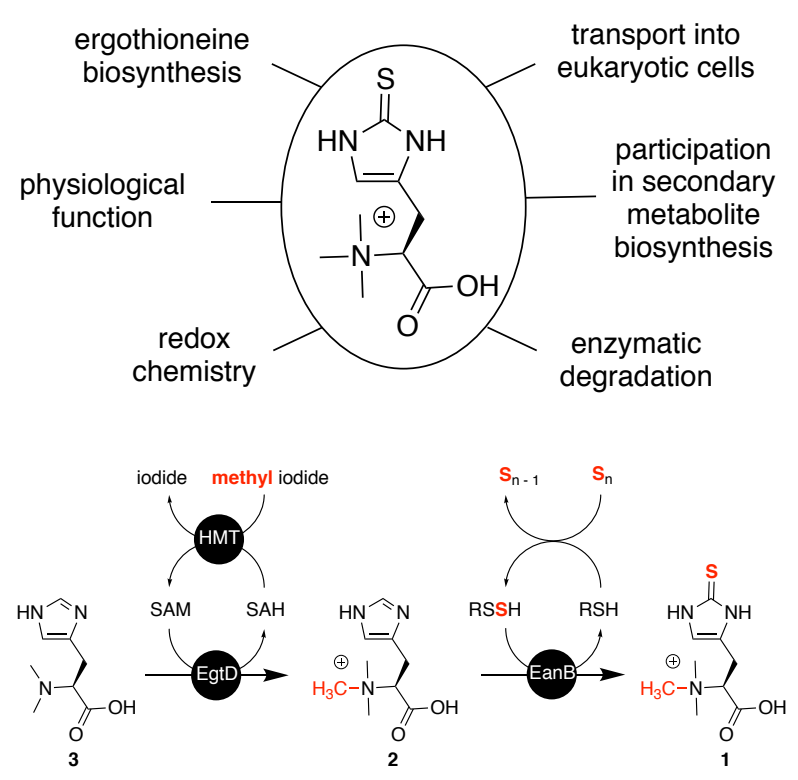
Isotope labeling is a powerful approach to identify, localize and quantify specific metabolites within complex media. ${ }^{[9]}$ For example, labeling with radio-isotopes was an essential tool in the early phase of ergothioneine research. Administering ${ }^{14} \mathrm{C}$-labeled histidine to rats was used to demonstrate that animals by themselves do not produce ergothioneine. ${ }^{[10]}$ Labeling studies with ${ }^{35} \mathrm{~S}-$ or ${ }^{14} \mathrm{C}$ containing precursors was used to decipher the biosynthetic logic of ergothioneine production by mycobacteria and fungi. ${ }^{[1]}$ More recently, uniform stable isotope labelling in the context of untargeted metabolite profiling provided first evidence that cyanobacteria produce ergothioneine. ${ }^{[12]}$ This finding was confirmed later based on genetic and more analytical evidence. ${ }^{[3 a, 13]}$

Similar strategies may open new ways to examine the metabolic fate of ergothioneine in oxidatively stressed cells, to quantify the rate of ergothioneine degradation by pathogenic and commensal members of the human microbiome, or to identify new ergothioneine derivatives within complex media. Ergothioneine isotopologues are accessible via chemical synthesis. ${ }^{[14]}$ However, total synthesis with rare isotopes is often expensive and the production of radioactive compounds requires specific safety measures. As a more efficient alternative we developed an enzyme-catalyzed one-pot cascade reaction that produces ergothioneine from histidine, methyl iodide and elemental sulfur (Figure 1). This approach is particularly versatile because all starting materials are commercially available in various isotopic compositions. To develop this methodology we combined a system that enables preparative $\mathrm{N}$-methylation of histidine ${ }^{[15]}$ with a sulfur transferase $(\mathrm{EanB})$ that can attach sulfur onto the imidazole ring of $\mathrm{N} \alpha$ trmethylhistidine. ${ }^{[16]}$ As a demonstration of scope, we used this methodology to produce eight different ergothioneine isotopologues.

\section{Results and Discussion}

EanB-catalyzed sulfurization of TMH. The biosynthesis of ergothioneine can occur through different pathways. ${ }^{[3 b]}$ Mycobacteria require five enzymes to methylate histidine and to mediate $\mathrm{O}_{2}$-dependent transfer of a sulfur atom from cysteine to $\mathrm{N \alpha}$ trimethylhistidine (TMH, 2, Figure 1) ${ }^{[3 a]}$ Fungi and many proteobacteria require only three enzymes, but sulfur insertion occurs via the same oxidative chemistry. ${ }^{[3 b]}$ A number of strictly anaerobic bacteria an archaea produce ergothioneine just in two steps: trimethylation of histidine by a S-adenosylmethionine (SAM)-dependent methyltransferase, followed by sulfurization by an $\mathrm{O}_{2}$ independent sulfur transferase. ${ }^{[16 a]}$ The crystal structure of this sulfur transferase (EanB) from Chlorobium limicola revealed an active site cysteine (Cys412, Figure 2) that accepts an additional (sulfane ${ }^{[17]}$ sulfur atom to form a hydropersulfide. The sulfane sulfur is then transferred onto carbon 2 of the imidazole ring of the substrate TMH. ${ }^{[16 b]}$ By analogy to related sulfur transferases, ${ }^{[18]}$ we initially thought that a pyridoxal 5'-phosphate (PLP) dependent cysteine desulfurase is required to extract and transfer sulfur atoms from free cysteine to EanB. ${ }^{[16 a]}$ Upon further analysis we realized that soluble polysulfides generated from partially oxidized hydrogen disulfide can also serve as the primary sulfur donor. ${ }^{[16 b, 19]}$ This finding has been confirmed recently by an independent study. ${ }^{[20]}$ 
Measuring the rates of ergothioneine production at concentrations of $\mathrm{Na}_{2} \mathrm{~S}$ ranging from 1 to 50 mM showed that the enzyme reaches saturation at $5 \mathrm{mM}$ (entries $2-6$, Table 1). At this concentration EanB catalyzes TMH sulfurization at a rate of $1 \mathrm{~min}^{-1}$, which is two-fold faster that previously determined as maximal velocity under single turnover conditions (entry 1$){ }^{[16]}$ It is possible that attachment of additional sulfur atoms to cysteine residues outside of the catalytic center increase the activity of this enzyme. Despite the comparatively low turnover rate, EanB may be useful for preparative applications because it is remarkably robust. In a test reaction containing $50 \mathrm{mM} \mathrm{Na}_{2} \mathrm{~S}, 4.4 \mathrm{uM}$, EanB completely converted $2 \mathrm{mM}$ of TMH to ergothioneine, corresponding to nearly 500 turnovers per enzyme (Figure S4).

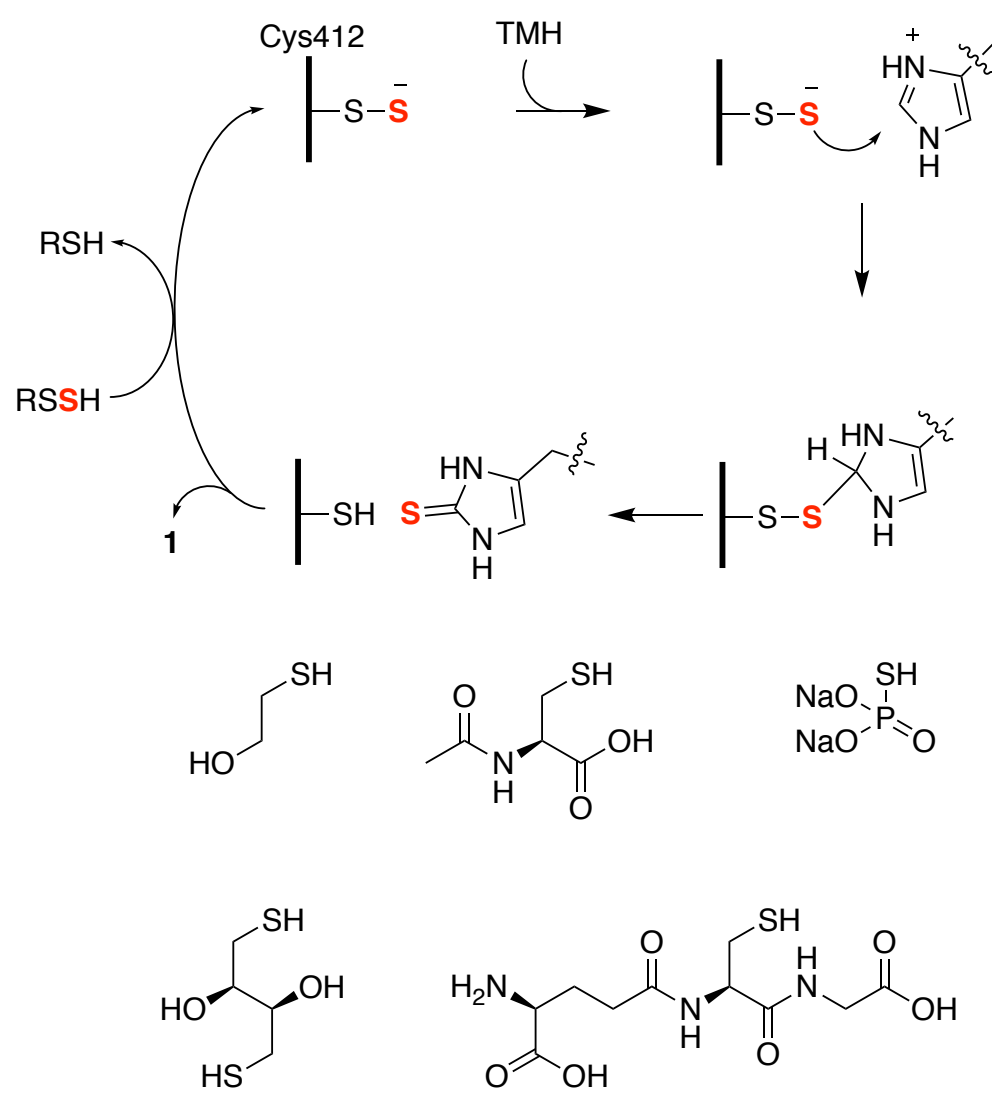

Figure 2. Top: Mechanism of EanB-catalyzed sulfurization of TMH. An active site cysteine (Cys412) accepts a sulfur atom from a hydropersulfide (R-S$\mathrm{SH})$ or a low weight polysulfide (R-Sn-SH). The sulfane sulfur in the active site of EanB is added to the imidazole ring of TMH in the second half-reaction. ${ }^{[16]}$ Bottom: Tested sulfur carriers (R-SH): $\beta$-mercapto ethanol ( $\beta \mathrm{ME}$ ), N-acetyl cysteine (NAC), thiophosphate, 1,4-dithiothreitol (DTT) and glutathione (GSH). 
Table $1^{[a]}$ Rates of EanB-catalyzed sulfurization of TMH using $\mathrm{Na}_{2} \mathrm{~S}$ and $\mathrm{S}_{8}$ as sulfur donor

\begin{tabular}{c|l|c}
\hline entry & Conditions & Rate, min $^{-1}$ \\
\hline 1 & EanB (single turnover) & 0.6 \\
3 & EanB $+1 \mathrm{mM} \mathrm{Na}_{2} \mathrm{~S}$ & 0.04 \\
4 & EanB $+2.5 \mathrm{mM} \mathrm{Na}_{2} \mathrm{~S}$ & 0.13 \\
5 & EanB $+5 \mathrm{mM} \mathrm{Na}_{2} \mathrm{~S}$ & 0.95 \\
6 & EanB $+50 \mathrm{mM} \mathrm{Na}_{2} \mathrm{~S}$ & 1.0 \\
7 & EanB $+10 \mathrm{mg} \mathrm{S}_{8}$ & 1.2 \\
8 & EanB $+10 \mathrm{mg} \mathrm{S}_{8}+1 \mathrm{mM} \mathrm{Na}_{2} \mathrm{~S}$ & 0.98 \\
\hline
\end{tabular}

$\overline{[a]}$ Observed rates for EanB-catalyzed conversion of TMH to ergothioneine. Reaction conditions: $100 \mathrm{mM}$ phosphate buffer $\mathrm{pH} 8.0,100 \mathrm{mM} \mathrm{NaCl}, 0.5 \mathrm{mM}$ TMH, $2.2 \mu \mathrm{M}$ EanB, $\mathrm{pH} 8.0,25^{\circ} \mathrm{C}$. The corresponding [1] vs. time plots are shown in Figure S3. The production rates are associated with an estimated error of less than $20 \%$ of the given values.

For preparative applications $\mathrm{Na}_{2} \mathrm{~S}$ is not an optimal reagent because it is toxic, hygroscopic and prone to oxidation upon storage. Therefore, we explored whether elemental sulfur $\left(\mathrm{S}_{8}\right)$ - a more stable and cheaper form of sulfur - could also serve as a reagent for the EanB reaction. Indeed, reactions containing suspended $\mathrm{S}_{8}$ in addition to $1 \mathrm{mM} \mathrm{Na} \mathrm{S}_{2}$ produced ergothioneine at the same rate as a reaction containing $50 \mathrm{mM} \mathrm{Na}_{2} \mathrm{~S}$ (entry 8, Table 1). By contrast, reactions containing either $\mathrm{S}_{8}$ or $1 \mathrm{mM} \mathrm{Na}_{2} \mathrm{~S}$ were 100 - and 25fold less productive (entries 7 and 2). These observations suggest that $\mathrm{Na}_{2} \mathrm{~S}$ acts as a sulfur mobilizing catalyst. As a drawback, mixing $\mathrm{Na}_{2} \mathrm{~S}$ with $\mathrm{S}_{8}$ makes the origin of the sulfur in ergothioneine ambiguous. In search for sulfur carriers that avoid this problem we compared the rates of TMH consumption and ergothioneine production in reactions containing $\mathrm{S}_{8}, 1 \mathrm{mM} \mathrm{TMH}, 10 \mathrm{uM}$ EanB and $10 \mathrm{mM}$ of either $\beta$-mercaptoethanol ( $\beta \mathrm{ME}), \mathrm{N}$-acetyl cysteine (NAC), dithiothreitol (DTT), glutathione (GSH) or one of the non-thiol reducing agents thiophosphate $\left(\mathrm{Na}_{3} \mathrm{PO}_{3} \mathrm{~S}\right)$ and sodium borohydride $\left(\mathrm{NaBH}_{4}\right.$, Figure S5). From this comparison GSH and $\beta \mathrm{ME}$ emerged as the most efficient sulfur transfer reagents. $\beta \mathrm{ME}$ was selected for further protocol development because of its relatively low cost. In the presence of $\mathrm{S}_{8}$ and $0.3-10 \mathrm{mM} \beta \mathrm{ME}$ the rate of EanB catalyzed sulfurization of TMH is dependent on $[\beta \mathrm{ME}]$. In the absence of elemental sulfur no ergothioneine formed, showing that $\beta \mathrm{ME}$ is not consumed as a sulfur donor (Figure S6).

Ergothioneine biosynthesis from histidine, methyl iodide and $\mathbf{S}_{\mathbf{8}}$. In a next step we combined this simplified system for TMH sulfurization with an enzyme cascade that produces TMH from histidine or N $\alpha$-dimethlyhistidine (DMH). We have previously shown that the histidine-specific methyltransferase EgtD from Mycolicibacterium smegmatis catalyzes three cooperative 
methylation steps converting histidine to TMH. ${ }^{[15,21]}$ Each methylation converts one equivalent of SAM to S-adenosylhomocysteine (SAH). In the cascade reaction SAH is methylated back to SAM by a halide methyltransferase (HMT) which uses methyl iodide as methyl donor (Figure 1). This system is remarkably efficient. A reaction containing $20 \mathrm{uM} \mathrm{HMT,} 20 \mathrm{uM}$ EgtD, $40 \mathrm{uM} \mathrm{SAH,} 3 \mathrm{mM}$ DMH, $3.3 \mathrm{mM}$ MeI in phosphate buffer at $\mathrm{pH} 8$ converted $3 \mathrm{mM}$ DMH to TMH within one hour (Figure 3, left). A similar reaction containing $3 \mathrm{mM}$ histidine and $10 \mathrm{mM}$ MeI was complete within $8 \mathrm{~h}$. Addition of $20 \mathrm{uM}$ EanB, $10 \mathrm{mM} \beta \mathrm{ME}$, and $1 \mathrm{mg} \mathrm{S}_{8}$ to these solutions converted all TMH to ergothioneine within seven days (Figure 3, right). Removal of the enzymes by heat denaturation and filtration produced a solution containing ergothioneine iodide and $\beta \mathrm{ME}$ dissolved in phosphate buffer. The final product was purified by ion exchange-HPLC (IE-HPLC, Figure S7) and analyzed by high resolution electrospray ionization mass spectrometry (HR-ESI-MS) and ${ }^{1} \mathrm{H}$ NMR (Figure S8).
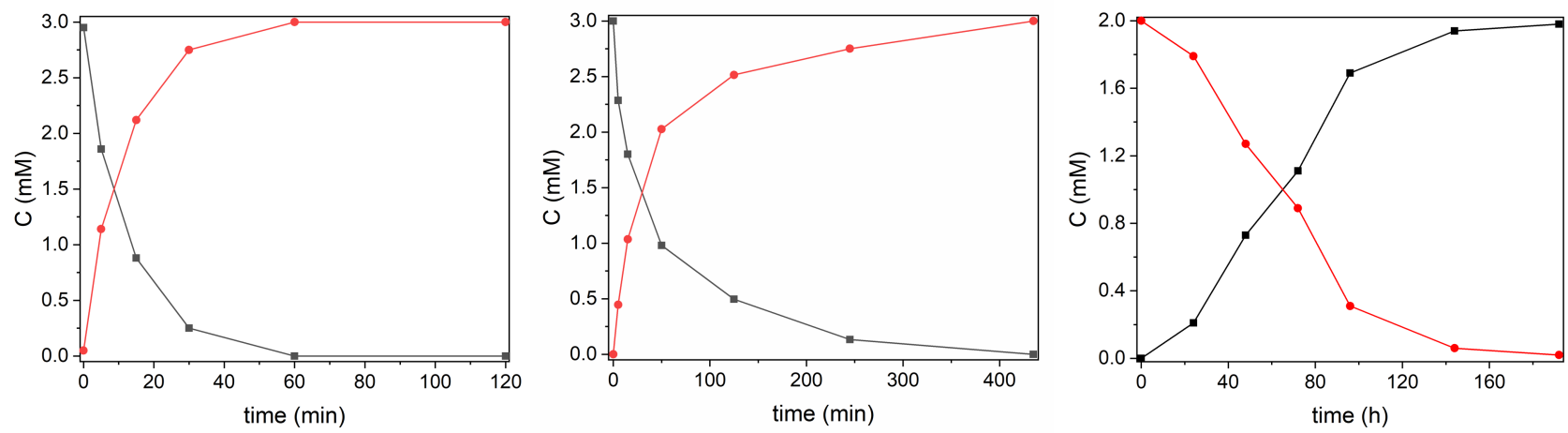

Figure 3. Methylation of DMH (left, black) or L-histidine (middle, black) to TMH (red). Reaction conditions: 20 uM HMT, 20 uM EgtD, 40 uM SAH, 3 mM DMH (or $3 \mathrm{mM}$ histidine), 1.1 equivalents (or 3.3 equivalents) of MeI in $20 \mathrm{mM}$ phosphate buffer, $\mathrm{pH} 8,25^{\circ} \mathrm{C}$. Right: Sulfurization of TMH (red) to ergothioneine (black): Reaction conditions: $20 \mathrm{uM} \mathrm{EanB,} 10 \mathrm{mM} \beta \mathrm{ME}, \sim 0.5-1 \mathrm{mg} \mathrm{S} \mathrm{S}_{8}, 2 \mathrm{mM} \mathrm{TMH}$ in phosphate buffer, $\mathrm{pH} 8,25^{\circ} \mathrm{C}$. The reactions were followed by IE-HPLC.

Production and characterization of ergothioneine isotopologues. The same procedure was applied to generate a number of exemplary ${ }^{2} \mathrm{H},{ }^{13} \mathrm{C}$ and ${ }^{15} \mathrm{~N}$ labelled ergothioneine isotopologues with ionic masses (m/z) ranging from 230 to 240 (Figure 4 ). The synthesis of derivatives 4 and 5 required deuterated or ${ }^{13} \mathrm{C}$-labelled methyl iodide as reagents. Derivative $\mathbf{6}$ was produced from deuterated DMH that was preparted by reductive amination with deuterated formaldehyde and sodium cyanoborodeuteride $\left(\mathrm{NaBD}_{3} \mathrm{CN}\right)$. Each of the three derivatives were isolated by IE-HPLC with yields of around 70 \% (Figure S9, Table S2). Derivatives 7 and 8 were obtained with similar yields using elemental ${ }^{33} \mathrm{~S}$ and ${ }^{34} \mathrm{~S}$. HR-ESI-MS analysis confirmed the faithful incorporation of the heavy sulfur isotopes, without competition by ${ }^{32} \mathrm{~S}$ derived from the sulfur carrier $\beta \mathrm{ME}$. Because this one-pot reaction requires minimal manipulation, the same protocol should also be applicable to the preparation of radio-isotopologues without production of excessive radioactive waste. It is also worth noting that that the reaction conditions can be optimized to completely consume MeI or 
$\mathrm{DMH}$, ensuring that most of the ${ }^{3} \mathrm{H}$ or ${ }^{14} \mathrm{C}$ labels are incorporated into the probe. Excess elemental sulfur can be removed simply be centrifugation. Efficient depletion of the hot reagents may render post-synthesis purification unnecessary.

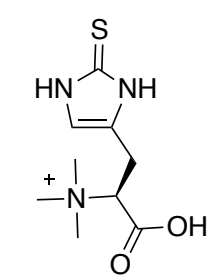

1: $\mathrm{m} / \mathrm{z}$ calc. 230.0963

$\mathrm{m} / \mathrm{z}$ obs. 230.0957

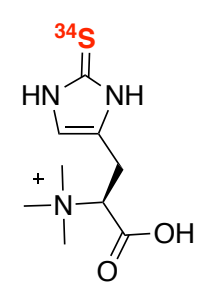

7: 232.0921 232.0916

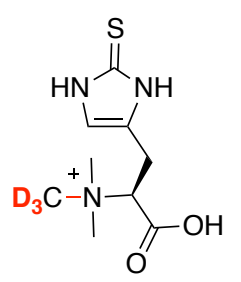

4: 233.1152 233.1143

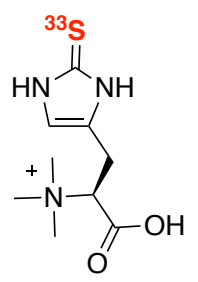

8: 231.0957 231.0947

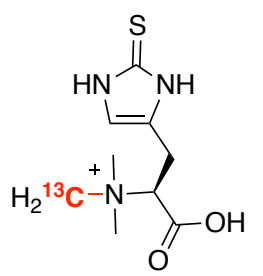

5: 231.0997 231.0990

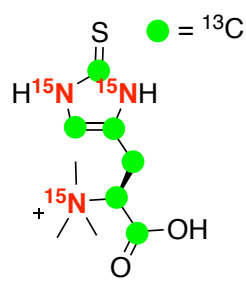

9: 239.1076 239.1072

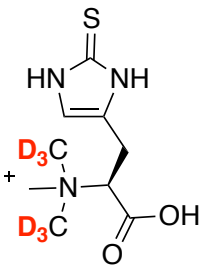

6: 236.1340 236.1331

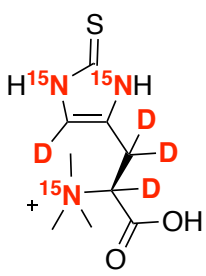

10: 237.1125 237.1119

Figure 4. Ergothioneine isotopologues synthesized by in vitro biocatalysis. Expected masses were calculated with enviPat. ${ }^{[22]}$

\section{CONCLUSSION}

In vitro biocatalysis offers new options in the generation of isotopically labelled natural products. In this report we describe a onepot cascade reaction that produces ergothioneine from commercially available isotope reagents. This synthesis entails two enzyme catalyzed group-transfer steps that are driven by two cyclic regeneration reactions. In the first reaction the methyl donor SAM is regenerated by HMT from SAH and MeI. In the second reaction the hydropersulfide in EanB is regenerated by $\beta$ ME that delivers sulfane sulfur from $\mathrm{S}_{8}$. The enzymes required for this process can be accessed by standard protocols for recombinant protein production and purification. The resulting ergothioneine derivatives can be purified efficiently by IE-HPLC. Given the ease of implementation we anticipate that the protocol described here will prove useful as a tool in the many approaches to study the intriguing and multifaceted biology of ergothioneine.

\section{ACKNOWLEDGMENT}

This project was supported by the NCCR for Molecular Systems Engineering and by the "Professur für Molekulare Bionik". 


\section{REFERENCES}

[1] aB. Halliwell, I. K. Cheah, R. M. Y. Tang, FEBS Lett. 2018, 592, 3357 - 3366; bI. Borodina, L. C. Kenny, C. M. McCarthy, K. Paramasivan, E. Pretorius, T. J. Roberts, S. A. van der Hoek, D. B. Kell, Nutr Res Rev. 2020, 13, 1 - 28; cB. N. Ames, Proc Natl Acad Sci US A. 2018, 115, 10836 - 10844.

[2] aD. Gründemann, S. Harlfinger, S. Golz, A. Geerts, A. Lazar, R. Berkels, N. Jung, A. Rubbert, E. Schoemig, Proc. Natl. Acad. Sci. U. S. A. 2005, 102, 5256-5261; bJ. Tschirka, M. Kreisor, J. Betz, D. Gründemann, Drug Metab Dispos. 2018, 46, 779 785; cR. A. J. Tucker, I. K. Cheah, B. Halliwell, Biochem. Biophys. Res. Commun. 2019, 513, 22 - 27.

[3] aF. P. Seebeck, J. Am. Chem. Soc. 2010, 132, 6632-6633; bA. R. Stampfli, W. Blankenfeldt, F. P. Seebeck, Curr. Opin. Struct. Biol. 2020, 65, 1 - 8.

[4] aB. M. Cumming, K. C. Chinta, V. P. Reddy, A. J. C. Steyn, Antioxid. Redox Signal. 2018, 28,431 - 444; bA. M. Gamage, C. Liao, I. K. Cheah, Y. Chen, D. R. X. Lim, J. W. K. Ku, R. S. L. Chee, M. Gengenbacher, F. P. Seebeck, B. Halliwell, Y. H. Gan, FASEB J. 2018, 11:fj201800716.

[5] Q. Zhao, M. Wang, D. Xu, Q. Zhang, W. Liu, Nature 2015, 518, 115 - 119.

[6] aI. K. Cheah, R. M. Y. Tang, T. S. Z. Yew, K. H. C. Lim, B. Halliwell, Antioxid. Redox Signal. 2017, 26, 193 - 206 ; bL. Servillo, D. Castaldo, R. Casale, N. D'Onofrio, A. Giovane, D. Cautela, M. L. Balestrieri, Free Radic. Biol. Med. 2015, 79, 228 -236 .

[7] M. Oumari, B. Goldfuss, C. Stoffels, H. G. Schmalz, D. Gründemann, Free Radic Biol Med. 2019, 134,498 - 504.

[8] aH. Muramatsu, H. Matsuo, N. Okada, M. Ueda, H. Yamamoto, S. Kato, S. Nagata, Appl Microbiol Biotechnol. 2013, 97,5389 - 5400; bA. Maurer, F. Leisinger, D. Lim, F. P. Seebeck, Chemistry 2019, 25, 10298 - 10303; cH. Muramatsu, H. Miyaoku, S. Kurita, H. Matsuo, T. Kashiwagi, C. S. Kim, M. Hayashi, H. Yamamoto, S. I. Kato, S. Nagata, J. Biochem. 2020, 167,333 341.

[9] J. Atzrodt, V. Derdau, W. J. Kerr, M. Reid, Angew Chem Int Ed Engl 2018, 57, 1758 - 1784.

[10] D. B. Melville, W. H. Horner, C. C. Otken, M. L. Ludwig, J. Biol. Chem. 1955, 213, 61-68.

[11] aJ. Wildy, H. Heath, Biochem. J. 1957, 65, 220 - 222; bJ. Wildy, H. Heath, Biochem. J. 1956, 64, 612 - 620; cD. S. GENGHOF, O. Van Damme, J. Bacteriol. 1964, 87, 852 - 862; dD. B. Melville, S. Eich, M. L. Ludwig, J. Biol. Chem. $1957,224,871$ - 877.

[12] R. Baran, B. P. Bowen, N. J. Bouskill, E. L. Brodie, S. M. Yannone, T. R. Northen, Anal. Chem. 2010, 82,9034 - 9042.

[13] C. Pfeiffer, B. Surek, E. Schöming, D. Gründemann, Food Chem. 2011, 129, 1766 - 1769.

[14] aI. Erdelmeier, S. Daunay, R. Lebel, L. Farescour, J. C. Yadan, Green Chemistry 2012, 14, 2256 - 2265; bJ. Xu, J. C. Yadan, J. Org. Chem. 1995, 60, 6296 - 6301; cP. L. Khonde, A. Jardine, Org. Biomol. Chem. 2015, 13, 1415 - 1419.

[15] C. Liao, F. P. Seebeck, Nat. Catal. 2019, 2, 696 - 701.

[16] aR. Burn, L. E. Misson, M. Meury, F. P. Seebeck, Angew Chem Int Ed Engl. 2017, 56, 12508 - 12511; bF. Leisinger, R. Burn, M. Meury, P. Lukat, F. P. Seebeck, J. Am. Chem. Soc. 2019, 141, 6906 - 6914.

[17] T. JI, Nat Rev Mol Cell Biol. 2012, 13, 803.

[18] J. U. Dahl, A. Urban, A. Bolte, P. Sriyabhaya, J. L. Donahue, M. Nimtz, T. J. Larson, S. Leimkühler, J Biol Chem. 2011, 286, $35801-35812$.

[19] R. Greiner, Z. Pálinkás, K. Bäsell, D. Becher, H. Antelmann, P. Nagy, T. P. Dick, Antioxid Redox Signal. 2013, 19 , 1749 1765.

[20] R. Cheng, L. Wu, R. Lai, C. Peng, N. Naowarojna, W. Hu, X. Li, S. A. Whelan, N. Lee, J. Lopez, C. Zhao, Y. Yong, J. Xue, X. Jiang, M. W. Grinstaff, Z. Deng, J. Chen, Q. Cui, J. Zhou, P. Liu, ACS Catal. 2020, 10, 8981 - 8994.

[21] L. Misson, R. Burn, A. Vit, J. Hildesheim, M. Beliaeva, W. Blankenfeldt, F. P. Seebeck, ACS Chem Biol. 2018, 13 , 1333 1342 .

[22] M. Loos, C. Gerber, F. Corona, J. Hollender, H. Singer, Anal. Chem. 2015, 87, 5738 - 5744. 